\section{Properties of Plastic, Peat, and Processed Poultry Feather Fiber Growing Containers}

\author{
Michael R. Evans ${ }^{1}$ and Douglas Karcher ${ }^{2}$ \\ Department of Horticulture, University of Arkansas, Fayetteville, AR 72701
}

Additional index words. pots, paper pots, water usage, decomposition

Abstract. When the substrate surface and drainage holes of feather fiber, peat, and plastic containers were sealed with wax, hyperbolic growth curves were good fits to cumulative water loss on a per container and per $\mathrm{cm}^{2}$ basis, with $R^{2}$ values ranging from 0.88 to 0.96 . The effect of container type was significant as the differences in asymptotic maximum water loss $(\max )$ values for all container pairs were significant at $P<0.05$ for both water loss per container and water loss per $\mathrm{cm}^{2}$. The predicted total water loss for peat containers was $\approx 2.5$ times greater than feather containers, and the predicted water loss per $\mathbf{c m}^{2}$ for the peat container was $\approx 3$ times greater than feather containers. Vinca [Catharanthus roseus $(\mathrm{L}$.) G. Don.] 'Cooler Blush' and impatiens (Impatiens walleriana Hookf.) 'Dazzler Rose Star' plants grown in feather and peat containers required more water and more frequent irrigations than those grown in plastic containers. However, plants grown in feather containers required less water and fewer irrigations than plants grown in peat containers. The surface area of containers covered by algal or fungal growth was significantly higher on peat containers than on feather containers. No fungal or algal growth was observed on plastic containers. Additionally, primarily algae were observed on peat containers whereas most discoloration observed on feather containers was due to fungal growth. Dry feather containers had a higher longitudinal strength than dry plastic containers but a lower longitudinal strength than dry peat containers. Wet feather containers had higher longitudinal strength than wet peat containers but a similar longitudinal strength as wet plastic containers. Dry feather and plastic containers had similar lateral strengths and both had significantly higher lateral strength than dry peat containers. Wet feather containers had significantly lower lateral strength than wet plastic containers but had higher lateral strength than wet peat containers. Dry and wet plastic containers had higher punch strength than wet or dry peat and feather containers. Dry peat containers had significantly higher punch strength than dry feather containers. However, wet feather containers had significantly higher punch strength than wet peat containers. Decomposition of peat and feather containers was significantly affected by container type and the species grown in the container. When planted with tomato (Lycopersicum esculentum L.) 'Better Boy', decomposition was not significantly different between the peat and feather containers. However, when vinca and marigold (Tagetes patula L.) 'Janie Bright Yellow' were grown in the containers, decomposition was significantly higher for feather containers than for peat containers. Therefore, containers made from processed feather fiber provided a new type of biodegradable container with significantly improved characteristics as compared to peat containers.

Greenhouse and containerized nursery crops are usually grown in plastic containers of varying sizes, depending upon the crop species and targeted market. Although plastic containers are strong and can be formed to essentially any size, shape, or color, they have two significant limitations. When grown in plastic containers, roots tend to circle the outer perimeter of the root ball once contact with the container is made. The roots circle the root ball, which can result in reduced growth and survival of plants when transplanted into the field (Appleton, 1993; Struve, 1993). Plastic containers also present a significant disposal issue for the horticulture industry. The disposal of used plastic containers is especially acute in the annual and perennial bedding plant markets,

Received for publication 11 Feb. 2003. Accepted for publication 9 Sept. 2003. This project was supported by Tyson Foods, Springdale, Ark., and the Arkansas Agricultural Research and Extension Service.

${ }^{1}$ Associate professor.

${ }^{2}$ Assistant professor. where relatively small plants are produced in large numbers. Growers may encounter disposal issues, particularly if plant materials are not sold during a season. Consumers and landscapers must remove plants from the plastic containers and are likewise left with containers for disposal.

Numerous attempts have been made to produce biodegradable containers that can be planted directly into the soil, thus reducing transplant shock and eliminating the need for disposal (Gayed, 1971; Lahde and Kinnonen, 1974; Mrazek, 1986). Typically, these have been made of peat, paper, or coir fiber. The most common biodegradable container has been the peat container. Although referred to as peat containers, they are typically made from a combination of peat and waste wood pulp or paper. Peat containers have significant disadvantages as compared to plastic containers. Peat containers are more expensive than plastic ones and break or tear easily when wet, making them difficult to handle. Plants grown in peat containers require more water than those grown in plastic containers, and algae often grows on the outside walls of peat containers, making them unsightly and difficult to handle. Peat containers are hydrophilic and, when planted into the field, they tend to wick water from the soil. Because this wicking allows the peat container wall to dry, the container can act as a significant impediment to root growth under certain conditions. Additionally, although biodegradable, the peat containers decompose very slowly and often must be removed from the soil and disposed of when the planting areas are replanted.

Poultry feathers are a significant waste component produced by the meat-processing industry. Depending upon location, feathers may be dried and ground for use in fertilizers (Choi and Nelson, 1996a, 1996b; Hadas and Kautsky, 1994) and animal feeds (Brown and Pate, 1997; Moritz and Latshaw, 2001; Palmquist et al., 1993), burned, or landfilled. Being made of the protein keratin, feathers are strong, biodegradable, and contain $\approx 15 \% \mathrm{~N}$ by weight (Hadas and Kautsky, 1994).

In cooperation with Tyson Foods (Springdale, Ark.), we developed a biodegradable container made primarily from processed waste poultry feather fiber (feather). Our objective in this research was to evaluate the performance characteristics, namely water usage, container strength, algal and fungal growth on container walls, and degree of decomposition of plastic, peat, and feather containers.

\section{Materials and Methods}

Waterloss through walls of plastic, peat, and feather containers. Plastic containers [Dura-Pot \#350-S (Hummert International, St. Louis)] were made of vacuum-formed black plastic. Plastic containers were $8.2 \mathrm{~cm}$ tall, and had top and bottom widths of $8.2 \mathrm{~cm}$. Plastic containers had a volume of $370 \mathrm{~mL}$. Peat containers (Jiffy Group International, Kristiansand, Norway) were $8.2 \mathrm{~cm}$ tall and had top and bottom widths of 8.0 and $5.2 \mathrm{~cm}$, respectively. Peat containers had a volume of $335 \mathrm{~mL}$. Although referred to as peat containers, these containers were made of $15 \%$ peat and $85 \%$ paper $(\mathrm{w} / \mathrm{w})$. The feather container design and composition were selected from 12 formulations evaluated prior to selection of the final test product for this study. The feather container (Tyson Foods) was composed of $85 \%$ processed feather fiber (feather) and $15 \%$ kraft paper (w/w). Feather containers were $8.3 \mathrm{~cm}$ tall with top and bottom widths of 8.1 and $5.5 \mathrm{~cm}$, respectively. Feather containers had a volume of $350 \mathrm{~mL}$.

Plastic, peat, and feather containers were filled with $330 \mathrm{~mL}$ of the sphagnum peat, composted bark, and perlite-based SB300 substrate (SunGro Horticulture, Bellevue, Wash.). The substrate was top watered to saturate the media and allow water to flow from the drainage holes of the containers. Water was applied so as to not saturate the peat and feather container walls. After drainage ceased, the drainage holes of the containers and the substrate surface were sealed with paraffin wax (Fisher Scientific, Fair Lawn, N.J.). The containers were weighed and placed in a glass-glazed greenhouse. Greenhouse air 
temperature was maintained between 20 and $23{ }^{\circ} \mathrm{C}$. Plants were grown under ambient light levels (458-635 $\mu \mathrm{mol} \cdot \mathrm{s}^{-1} \cdot \mathrm{m}^{-2}$ at 12:00 $\mathrm{HR}$ ). Containers were weighed at 24-h intervals for $7 \mathrm{~d}$ to determine water loss through the container walls. Because small differences in container dimensions existed, water loss was expressed on a total container basis as well as per $\mathrm{cm}^{2}$ of surface area. The experimental design was a completely randomized design with five replications of each container.

Upon plotting cumulative water loss over time for each pot type, a hyperbolic growth function $[\mathrm{cwl}=(\max \cdot d a y s) /(K+$ days $)]$ was chosen as a model, where $c w l$ was cumulative water loss, max was the asymptotic maximum water loss for each pot, days was the number of days following saturation, and $K$ was the number of days at which one-half maximum water loss is reached. The effect of container type on water loss was considered significant when either the max or $K$ parameters between a pair of containers were significantly different $(P<0.05)$ according to a $t$-test. The nonlinear regression analysis of the cumulative water loss data was performed using GraphPad Prism version 3.00 for Windows, GraphPad Software, San Diego.

Water requirement of plants grown in plastic, peat, and feather containers. All experimental protocol were as described for the first experiment except where indicated. Fourweek-old 'Dazzler Rose Star' impatiens and 'Cooler Blush' vinca plugs (seedlings in size 288 plug trays with cell volume of $5 \mathrm{~mL}$ ) were transplanted into the plastic, peat, and feather containers filled with $330 \mathrm{~mL}$ of substrate. Plants were placed in a glass-glazed greenhouse, and the substrate was drenched with the fungicide Banrot (Scotts, Marysville, Ohio) according to label directions. Greenhouse air temperature was maintained between 20 and $28^{\circ} \mathrm{C}$. Plants were grown under ambient light levels $\left(450-640 \mu \mathrm{mol} \cdot \mathrm{s}^{-1} \cdot \mathrm{m}^{-2}\right.$ at 12:00 $\left.\mathrm{HR}\right)$.

Twenty-four hours after containers were drenched with fungicide, they were placed on drain trays so that any water leaching from the container could be measured and deducted from the volume applied. Plants were fertilized at each irrigation with $200 \mathrm{~mL}$ of solution containing $200 \mathrm{mg} \cdot \mathrm{L}^{-1} \mathrm{~N}$ using a $15-2.2-12.5$ water-soluble fertilizer (Excel 15-5-15 Cal Mag, Scotts). Each container was irrigated individually with $150 \mathrm{~mL}$ of solution as the surface of the substrate began to dry as determined visually. Trays were placed underneath the containers to collect drainage. Number of irrigations, average volume of water retained per irrigation, total water required during the production cycle, number of irrigations, and average interval between irrigations were determined. The experiment was terminated after 5 weeks.

The experimental design was a complete randomized block with 10 blocks and each container type appearing once per block. Container type served as the main treatment effect and each container was considered to be an independent experimental unit. An analysis of variance was conducted to determine if significant differences occurred among the container types. Where significant differences occurred, an LSD mean separation test $(\alpha=$ 0.05 ) was conducted to determine individual differences between means.

Algal and fungal growthon peat and feather containers. All experimental protocol were as described for the second experiment except where indicated. Four-week-old impatiens plugs were transplanted into plastic, peat, and feather containers filled with $330 \mathrm{~mL}$ of substrate. Plants were placed in a glass-glazed greenhouse on expanded metal benches without saucers underneath. Plants were overhead irrigated when the substrate surface of $\approx 25 \%$ of the containers was dry as determined visually. After 8 weeks, plants and soil were removed from the containers. Containers were dried and dissected into fractions that were discolored with fungal or algal growth, and that which was not discolored with algal or fungal growth. The total container surface area and the discolored portion of the container was determined using an area meter (LI-3000; LI-COR, Lincoln, Nebr.). The container surface area discolored with algae or fungi was expressed as a percentage of the total surface area.

The experimental design was a complete randomized block with eight blocks and each container type appearing once in a block. Container type served as the main treatment effect and each container was considered to be an independent experimental unit.

Comparison of container strength of dry and wet plastic, peat, and feather containers. All experimental protocol were as described for the first experiment except where indicated. Plastic, peat, and feather containers were subjected to increasing amounts of pressure using a texture analyzer (TAXT 2I; Texture Technologies, Scarsdale, N.Y.) to determine the initial breaking point of the containers as well as the amount of pressure required to completely crush the containers. Containers were crushed longitudinally (top to bottom with container set upright) and laterally (container laying on side with weight placed on the bottom edge of the container). Container wall strength was also examined by determining the amount of pressure required to cause an initial break in the container wall as well as to punch through the container wall using a round $0.5-\mathrm{cm}$ ball probe. Both unused dry and wet containers that had been saturated with water for $7 \mathrm{~d}$ were tested. Five replications of each container were tested. Container type served as the main treatment effect, and each container was considered to be an independent experimental unit.

Decomposition of paper and feather containers under field conditions. All experimental protocol were as described for the second experiment except where indicated. Threeweek-old 'Better Boy' tomato, 'Janie Bright Yellow' marigold, and 4-week-old 'Cooler Blush' vinca plugs were transplanted into peat and feather containers filled with substrate. Plants were placed into the greenhouse and allowed to grow for 5 weeks before being planted into the field.

The field soil type was a Captina silt loam, typic hapludult. Peat and feather containers were left intact and planted with $\approx 0.5 \mathrm{~cm}$ of the container rim above the soil line. Plants were grown under full sun and irrigated every other day using drip irrigation. Plants were fertilized using the slow-release fertilizer Osmocote Pro 19-2.2-6.6 (Scotts) at the rate of $10 \mathrm{~g}$ per plant. After 16 weeks, the root mass was removed from the soil, and any remaining container material was removed, dried, and weighed. The level of decomposition of the container was determined and expressed as a percentage of the original weight of the pot before planting. The experimental design was a complete randomized block with six blocks, with each container type and plant species appearing once in a block. Container type and plant species served as the main treatment effects, and each container was considered an independent experimental unit.

\section{Results and Discussion}

Water loss through walls of plastic, peat, andfeather containers. The hyperbolic growth curves were good fits to the cumulative water loss data on a per container (Fig. 1) and per $\mathrm{cm}^{2}$ (Fig. 2) basis, with $R^{2}$ values ranging from 0.88 to 0.96 . The effect of container type was significant since the differences in max values for all container pairs were significant at $P$ $<0.05$ for both water loss per container and water loss per $\mathrm{cm}^{2}$. The predicted total water loss for the peat container (Fig. 1) was $\approx 2.5$ times greater than the feather container, while no appreciable water was lost from the plastic container. The feather, peat, and plastic containers reached one-half total water loss $(\mathrm{K})$ at 2 , 6 , and $35 \mathrm{~d}$ following saturation, respectively. The predicted water loss per $\mathrm{cm}^{2}$ for the peat container (Fig. 2) was $\approx 3$ times greater than the feather container, while no appreciable water was lost from the plastic container. The feather, peat, and plastic containers reached one-half total water loss per $\mathrm{cm}^{2}(\mathrm{~K})$ at 2, 6, and $25 \mathrm{~d}$ following saturation, respectively.

On a total container and per $\mathrm{cm}^{2}$ basis, plastic containers lost very little water through the container walls. Feather containers, although losing more water through the container walls than plastic containers, lost significantly less water through the container walls than peat containers. The difference observed between feather and peat containers can be explained at least partially by the fact that peat containers, made primarily of paper, were hydrophilic, while feather fiber containers were generally hydrophobic and presented a more water resistant barrier.

Water requirement of plants grown in plastic, peat, and feather containers. For both vinca and impatiens, total volume of water used and total number of irrigations were significantly lower for plastic containers than for peat and feather ones (Table 1). For both species, total volume of water used and total number of irrigations were significantly lower for feather containers than for peat ones. Water retained per irrigation was significantly lower for plastic containers than for peat and feather ones. Water retained per irrigation was not significantly different between peat containers and feather ones. Average irrigation interval 


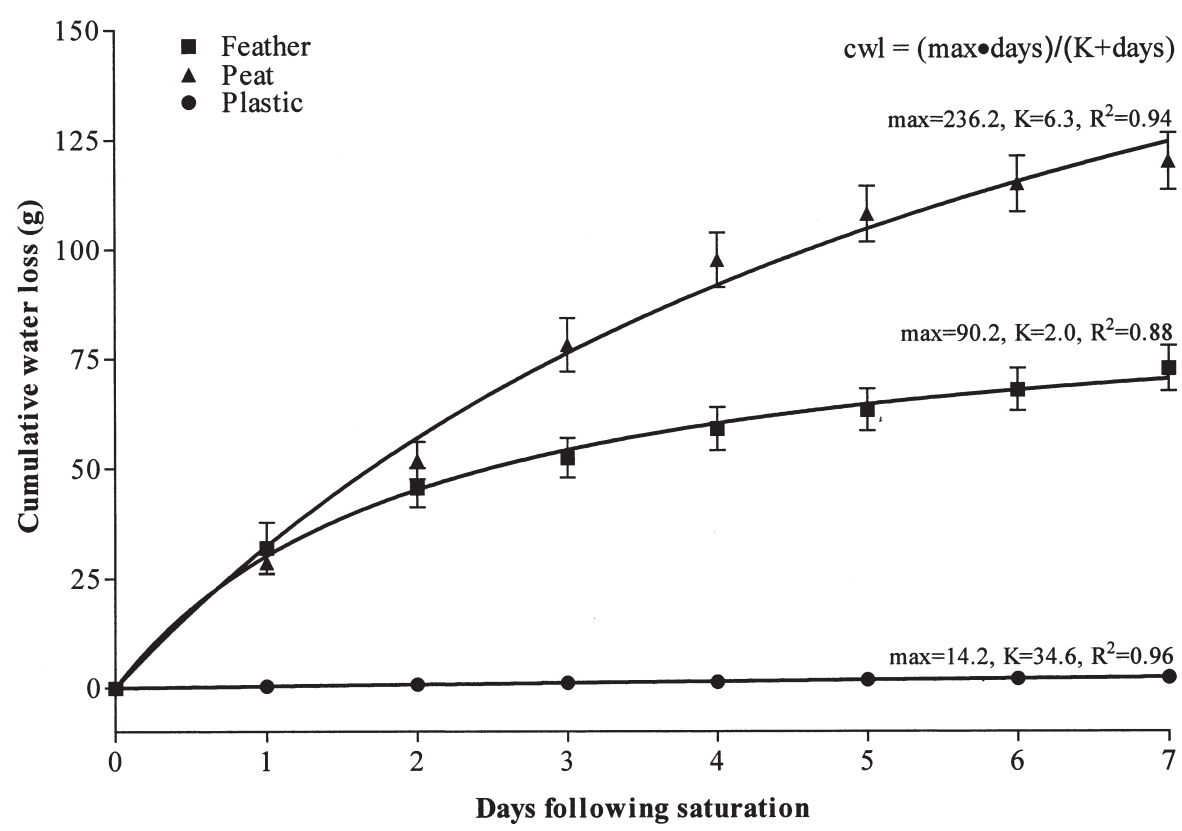

Fig. 1. Cumulative water loss per container for plastic, peat, and feather containers following saturation. $\mathrm{Cwl}=$ cumulative water loss, $\max =$ the asymptotic maximum water loss for each pot, days $=$ the number of days following saturation, and $\mathrm{K}=$ the number of days at which one-half maximum water loss is reached.

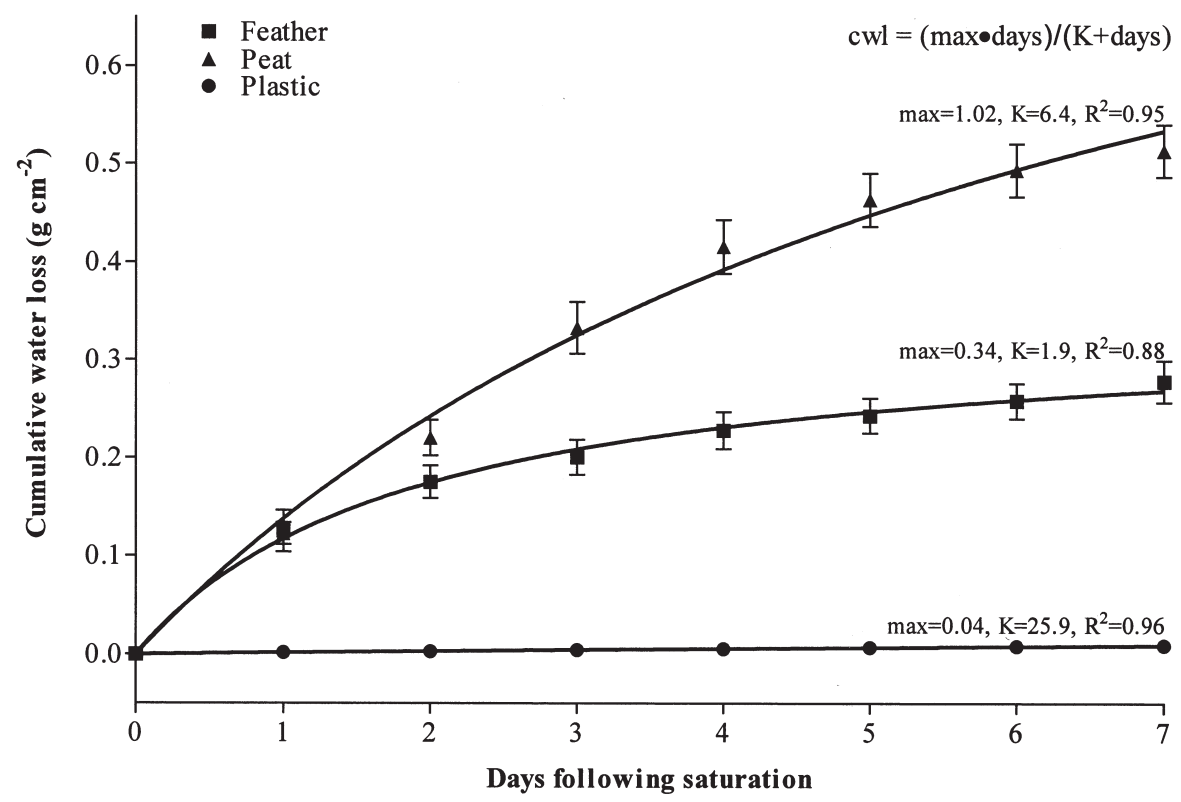

Fig. 2. Cumulative water loss per $\mathrm{cm}^{2}$ for plastic, peat and feather containers following saturation. $\mathrm{Cwl}=$ cumulative water loss, $\max =$ the asymptotic maximum water loss for each pot, days = the number of days following saturation, and $\mathrm{K}=$ the number of days at which one-half maximum water loss is reached.

Table 1. Water use, number of irrigations, water retained per irrigation and irrigation frequency for vinca and impatiens grown in plastic, peat and feather containers.

\begin{tabular}{|c|c|c|c|c|c|c|c|c|}
\hline \multirow[b]{2}{*}{ Container } & \multicolumn{2}{|c|}{$\begin{array}{c}\text { Total vol } \\
\text { of water used }(\mathrm{mL})\end{array}$} & \multicolumn{2}{|c|}{$\begin{array}{c}\text { Total no. } \\
\text { of irrigations }\end{array}$} & \multicolumn{2}{|c|}{$\begin{array}{l}\text { Water retained per } \\
\text { irrigation }(\mathrm{mL})\end{array}$} & \multicolumn{2}{|c|}{$\begin{array}{l}\text { Avg irrigation } \\
\text { interval }(\mathrm{d})\end{array}$} \\
\hline & Vinca & $\overline{\text { Impatiens }}$ & Vinca & Impatiens & Vinca & $\overline{\text { Impatiens }}$ & Vinca & Impatiens \\
\hline Plastic & 1943 & 1667 & 13.0 & 11.8 & 149.0 & 142.6 & 2.7 & 3.0 \\
\hline Peat & 4406 & 4327 & 26.6 & 26.6 & 166.0 & 162.8 & 1.3 & 1.3 \\
\hline Feather & 3112 & 2963 & 18.2 & 17.8 & 171.2 & 166.4 & 1.9 & 2.0 \\
\hline \multicolumn{9}{|l|}{ Significance } \\
\hline Container & $* * *$ & $* * *$ & $* * *$ & $* * *$ & $* * *$ & $* * *$ & $* * *$ & $* * *$ \\
\hline Block & NS & NS & NS & $* *$ & NS & $*$ & NS & $* *$ \\
\hline $\operatorname{LSD}(\alpha=0.05)$ & 257.2 & 171.6 & 1.4 & 1.0 & 7.5 & 6.7 & 0.2 & 0.2 \\
\hline
\end{tabular}

NS, ${ }^{* * *, * * *}$ Significant at $P=0.05,0.01$, or 0.001 , respectively. was significantly higher for plastic containers than for peat or feather ones, and was higher for feather containers than for peat ones.

Unlike peat and feather containers, plastic containers presented a nearly water impermeable barrier. Thus, evaporation from the substrate could only occur from the substrate surface in plastic containers, whereas evaporation could also occur through the wall of the peat and feather containers. Therefore, plants required more water and more frequent irrigations when grown in peat and feather containers than when grown in plastic ones. Plants grown in feather containers required less water and less frequent irrigations than plants grown in peat containers. This may have occurred because feather containers tended to be hydrophobic. whereas the peat ones were hydrophilic. Thus, although water could move through the walls of feather containers, it did so less readily than for peat ones. This conclusion is supported by the results from the first experiment, in which water loss through the container walls was demonstrated to be less for feather containers than for peat ones.

Algal and fungal growth on peat and feather containers. The surface area of containers covered by algal or fungal growth was significantly higher on peat containers than on feather ones (Table 2). No fungal or algal growth was observed on plastic containers. Additionally, primarily algae grew on peat containers, whereas most discoloration observed on feather containers was due to fungal growth. Peat containers were more easily wetted than feather ones when irrigated, due to their being hydrophilic, whereas feather containers were generally hydrophobic. Peat containers, being made primarily of paper, had a high lignin and cellulose concentration, whereas feather containers had a high nitrogen concentration (Hadas and Kautsky, 1994). These or other differences in the container wall chemistry may have impacted both the types of organisms and their amount of growth on the container walls.

Comparison of strength of dry and wet plastic, peat, and feather containers. Dry feather containers had a higher longitudinal break strength than dry plastic ones, but a lower longitudinal break strength than dry peat containers (Table 3). Wet feather containers had higher longitudinal break strength than wet peat containers but a similar longitudinal break strength as wet plastic containers. Dry feather and plastic containers had similar lateral break

Table 2. Algal and fungal growth on surface of plastic, peat, and feather containers.

\begin{tabular}{lc}
\hline Container & Coverage $(\%)^{\mathrm{z}}$ \\
\hline Plastic & 0 \\
Peat & 56.0 \\
Feather & 5.3 \\
Significance & \\
$\quad$ Container & $* * *$ \\
$\quad$ LSD $(\alpha=0.05)$ & 12.3 \\
\hline
\end{tabular}

${ }^{2}$ Percentage of container surface discolored by algae or fungi.

yPlastic containers showed no algal or fungal growth.

${ }^{* * *}$ Significant at $P=0.001$ 
strengths, and both had significantly higher lateral break strength than dry peat containers. Wet feather containers had significantly lower lateral break strength than wet plastic containers, but had higher lateral break strength than wet peat containers. Dry and wet plastic containers had higher punch break strength than wet or dry peat and feather containers. Dry peat containers had significantly higher punch break strength than dry feather containers. However, wet feather containers had significantly higher punch break strength than wet peat containers.

Dry feather containers had a higher longitudinal crush strength than dry plastic containers, but a lower longitudinal crush strength than dry peat ones (Table 3 ). Wet feather containers had higher longitudinal crush strength than wet peat containers and similar longitudinal crush strength as wet plastic ones. Dry feather containers had higher lateral crush strengths than dry feather containers but had significantly lower lateral crush strength than dry plastic ones. Wet feather containers had significantly lower lateral crush strength than wet plastic containers and similar lateral crush strength as wet peat containers. Dry feather containers had a lower punch through strength than dry peat and plastic containers. Dry and wet plastic containers had significantly higher punch through strength than either dry feather or dry peat containers. Wet feather containers had significantly higher punch through strength than wet peat containers and lower punch through strength than wet plastic containers.

When dry, peat containers were rigid and had a high level of strength. However, when wet, the paper component of the peat containers readily absorbed water and the peat containers became soft and significantly weaker than plastic or feather containers. When dry, feather containers had higher longitudinal and lateral strengths than plastic containers. Although weaker than plastic containers, wet feather containers maintained a higher wet strength than peat ones. This was at least partially due to the more rigid feather fibers in the walls of the feather containers as compared to the relatively weak and flexible paper fibers making up the peat containers. Additionally, since feather containers were generally hydrophobic and absorb water much less readily than the peat ones, water had less effect on container wall strength. Since containers filled with soil and in a greenhouse setting are usually wet, the higher wet strength of feather containers as compared to peat ones would be beneficial for users.

Decomposition of paper and feather containers under field conditions. Decomposition of peat and feather containers was significantly affected by container type and the species grown in the container (Table 4). A significant interaction between species and container was observed. For tomato, decomposition was not significantly different between the peat and feather containers. However, when vinca and marigold were grown in the containers, decomposition was significantly higher for feather containers than for peat containers. The interaction between species and container type may have been a function of several variables,

Table 3. Strength comparisons of wet and dry plastic, peat, and feather fiber containers.

\begin{tabular}{|c|c|c|c|c|c|c|c|}
\hline \multirow[b]{2}{*}{ Container $^{\mathrm{y}}$} & \multicolumn{4}{|c|}{ Initial break $(\mathrm{kg})^{\mathrm{z}}$} & \multicolumn{3}{|c|}{$\begin{array}{l}\text { Complete crush or } \\
\text { breakthrough }\left(\mathrm{kg} \cdot \mathrm{s}^{-1}\right)\end{array}$} \\
\hline & Condition & Longitudinal & Lateral & Punch & Longitudinal & Lateral & Punch \\
\hline Plastic & Dry & 5.8 & 3.8 & 17.3 & 42.4 & 31.9 & 19.6 \\
\hline Plastic & Wet & 5.9 & 3.6 & 17.1 & 42.5 & 31.6 & 19.6 \\
\hline Peat & Dry & 25.0 & 3.1 & 2.4 & 126.7 & 27.2 & 1.7 \\
\hline Peat & Wet & 3.4 & 1.5 & 0.6 & 18.0 & 12.4 & 0.5 \\
\hline Feather & Dry & 15.2 & 4.2 & 1.3 & 87.9 & 21.9 & 0.8 \\
\hline Feather & Wet & 5.3 & 2.2 & 1.1 & 36.2 & 13.1 & 0.8 \\
\hline \multicolumn{8}{|c|}{ Significance } \\
\hline Contain & & $* * *$ & $* * *$ & $* * *$ & $* *$ & $* * *$ & $* * *$ \\
\hline Conditic & & $* * *$ & $* * *$ & $*$ & $* * *$ & $* * *$ & NS \\
\hline Contain & ndition & $* * *$ & $* * *$ & NS & **** & $* *$ & NS \\
\hline $\operatorname{LSD}(\alpha=$ & & 1.7 & 0.7 & 0.4 & 17.7 & 3.7 & 0.3 \\
\hline
\end{tabular}

${ }^{\mathrm{z}}$ Longitudinal indicates pressure exerted on upright container from top to bottom. Lateral indicates pressure exerted against the side of the container at the bottom of the container. Punch indicates pressure required to break through the side wall of the container. Initial break indicates pressure required for first breakage of container or sidewall, and complete crush indicates total pressure required to crush container or break through container wall.

${ }^{y}$ Means for wet plastic were repeated for dry plastic, since dry plastic was impervious to water. Ns, ${ }^{* * *, * * *}$ Nonsignificant or significant at $P=0.05,0.01$, or 0.001 , respectively.

including differences in root growth. We observed that tomato plants had larger and more vigorous root systems than vinca and marigold. When containers were dug from the field, those grown with tomato had been physically broken apart more by the root system of the tomato. The physical breakage of the peat containers by the tomato root system may have resulted in an increased rate of decomposition and a loss in the significant difference between peat and feather containers observed when they were planted with vinca and marigold.

Peat containers were composed primarily of paper. The paper had a relatively high $\mathrm{C}: \mathrm{N}$ ratio as compared to feather containers, which are composed primarily of keratin and are typically $13 \% \mathrm{~N}$ by weight. The higher $\mathrm{N}$ levels of the feather containers may have supported a higher level of microbial growth, resulting in a higher degree of decomposition. With a decomposition level of $56.5 \%$ to $66.5 \%$ after 16 weeks in the field, little of the feather container remained; thus, removal would not be required before replanting of the location.

Processed feather fiber was successfully used as an alternative to peat and paper in making a biodegradable growing container. Containers made from the feather fiber had significant advantages over traditional peat containers, including requiring less water during crop production, higher wet strengths, less algae and fungal growth on the container walls, and a more rapid decomposition rate when planted into the field.

\section{Literature Cited}

Appleton, B.L. 1993. Using copper compounds to modify roots on container-grown trees. Combined Proc. Intl. Plant Prop. Soc. 43:376-379.

Brown, W.F. and F.M. Pate. 1997. Cottonseed meal or feather meal supplementation of ammoniated tropical grass hay for yearly cattle. J. Animal Sci. 75:1666-1673.

Choi, J.M. and P.V. Nelson. 1996a. Developing a slow-release nitrogen fertilizer from organic sources. II. Using poultry feathers. J. Amer. Soc. Hort. Sci. 121:634-638.

Choi, J.M. and P.V. Nelson. 1996b. Developing a
Table 4. Degree of decomposition of peat and feather containers under field conditions.

\begin{tabular}{llr}
\hline Species & Container & $\begin{array}{r}\text { Decomposition } \\
(\%)^{\mathrm{z}}\end{array}$ \\
\hline Tomato & Feather & 56.5 \\
Tomato & Peat & 63.0 \\
Vinca & Feather & 62.3 \\
Vinca & Peat & 9.5 \\
Marigold & Feather & 66.5 \\
Marigold & Peat & 26.5 \\
Significance & & \\
$\quad$ Species (S) & & $* *$ \\
Container $(C)$ & & $* * *$ \\
Block & & NS \\
S $\times$ C & & $* *$ \\
LSD $(\alpha=0.05)$ & & 21.6 \\
\hline 2Decomposition a percentage of weight loss after
\end{tabular}

${ }^{2}$ Decomposition as a percentage of weight loss after 16 weeks under field conditions.

NS, $* * * * * *$ Nonsignificant or significant at $P=0.01$ or 0.001 , respectively.

slow-release nitrogen fertilizer from organic sources. III. Isolation and action of a featherdegrading actinomycete. J. Amer. Soc. Hort. Sci. 121:639-643.

Gayed, S.K. 1971. Effect of transplanting tobacco seedlings in peat pots on plant vigor and on susceptibility to Thielaviopsis root rot. Can. Plant Dis. Survey 51(4):142-144.

Hadas, A. and L. Kautsky. 1994. Feather meal, a semi-slow-release nitrogen fertilizer for organic farming. Fert. Res. 38(2):165-170.

Lahde, E. and K. Kinnonen. 1974. The relationship between wall strength of paper and peat pots and the initial development of seedlings in northern Finland. Folia Forestalia 197:1-19.

Moritz, J.S. and J.D. Latshaw. 2001. Indicators of nutritional value of hydrolyzed feather meal. Poultry Sci. 80(1):79-86.

Mrazek. F. 1986. Comparative growth studies of plants in peat pots and with naked roots in an advanced plantation of douglas firs. Beitr. Forstwirtschaft 20(3):128-129.

Palmquist, D.M., M.R. Weisbjerg, and T. Hvelplund. 1993. Ruminal, intestinal and total disgestibilities of nutrients in cows fed diets high in fat and undegradable protein. J. Dairy Sci. 76:1353-1364.

Struve, D.K. 1993. Effect of copper-treated containers on transplant survival and regrowth of four tree species. J. Environ. Hort. 11:196-199. 